\title{
CACO-2 CELLS, BIOPHARMACEUTICS CLASSIFICATION SYSTEM (BCS) AND BIOWAIVER
}

\author{
Libuše Smetanová, Věra Štětinová, Zbyněk Svoboda, Jaroslav Květina
}

Institute of Experimental Biopharmaceutics, Joint Research Centre of PRO.MED.CS Praha a.s. and the Academy of Sciences of the Czech Republic, Heyrovského 1207, Hradec Králové, Czech Republic

\begin{abstract}
Summary: Almost all orally administered drugs are absorbed across the intestinal mucosa. The Caco-2 monolayers are used as an in vitro model to predict drug absorption in humans and to explore mechanism of drug absorption. The Caco- 2 cells are derived from a human colon adenocarcinoma and spontaneously differentiate to form confluent monolayer of polarized cells structurally and functionally resembling the small intestinal epithelium. For studying drug permeability, Caco-2 cells are seeded onto the Transwell inserts with semipermeable membrane and grown to late confluence (21 days). After determination of cell viability, the integrity of monolayer is checked by phenol red permeability and by ${ }^{14} \mathrm{C}-\mathrm{mannitol}$ permeability. The transport from apical to basolateral (AP-BL) and basolateral to apical (BL-AP) is studied by adding the diluted drug on the apical or basolateral side and withdrawing the samples from the opposite compartment, respectively, for HPLC analysis or liquid scintillation spectrometry. $\mathrm{Ca}^{2+}$ - free transport medium is used to determine paracellular component of the drug transport. On the basis of permeability and solubility, drugs can be categorized into four classes of Biopharmaceutics Classification System (BCS). For certain drugs, the BCS-based biowaiver approach can be used which enables to reduce in vivo bioequivalence studies.
\end{abstract}

Key words: Caco-2 cells; Intestinal transport; Permeability coefficient ( $\left.P_{\text {app }}\right)$; Biopharmaceutics Classification System (BCS); Biowaiver

\section{Introduction}

Many factors can affect the intestinal absorption rate of drugs, such as drug dissolution characteristics, luminal $\mathrm{pH}$, luminal contents and drug transport proteins (12). Good permeability through intestinal membranes leads to adequate systemic absorption. A cell model system, such as Caco-2 cells, with unusually high degree of differentiation together with the fact that these cells differentiate spontaneously in normal serum-containing cell culture medium, has become one of the most popular cell lines in studies of epithelial transport in vitro model (19) to predict drug absorption in humans, to explore mechanism of drug absorption and identify substrates or inhibitors of transporters (3). The good correlation between permeability across Caco-2 cell monolayer, solubility and oral bioavailability in humans has been demonstrated (30).

Amidon et al. (2) recognized the fundamental parameters controlling the rate and extent of oral drug absorption and devised Biopharmaceutics Classification System (BCS) that categorized drugs into four classes according to their solubility and permeability. The objective of the BCS is to predict in vivo pharmacokinetic performance of drug products from measurements of permeability and solubility (2).
The aim of this review is to provide an insight into the Caco-2 cells experiments, Biopharmaceutics Classification System and its use for BCS-based biowaiver approach, which enables to reduce in vivo bioequivalence studies, i.e., it may represent a surrogate for in vivo bioequivalence.

\section{Caco-2 cells}

\section{Caco-2 cells origin and morphology}

The Caco-2 cells line is a continuous line of heterogenous human epithelial colorectal adenocarcinoma cells, developed by the Sloan-Kettering Institute for Cancer Research through research conducted by Jorgen Fogh (16). Although cancerous in origin, Caco- 2 cells are unique in their ability to initiate spontaneous differentiation on reaching confluence under normal culture conditions (i.e., in presence of glucose and serum). Consequently, over a period of 20-30 days of postconfluent culture these cells gradually show a morphological polarity and levels of brush border hydrolases that are both comparable with those of mature intestinal absorptive cells. Beginning at confluence, the brush border develops progressively. From 5 to 20 days postconfluence, a gradual reduction of the surface occu- 
pied by each cell as well as the formation of more intimate intercellular junctions are noted. And in this time also the length and density of microvilli increase. After 30 day, the cell surface reaches a minimum and cells have a typical enterocyte-like morphology. When complete structural polarization is achieved ( 30 days), most of the cells have fully developed brush border with tall and regular microvilli uniformly distributed in a ruglike organized fashion (31). The morphological and ultrastuctural characterizations of Caco-2 cells show significant heterogeneity throughout the monolayer (31). Caco-2 cells have three distinctive states in culture: homogeneously undifferentiated (at subconfluence), heterogenously differentiated (0-20 day postconfluence), and homogenously polarized and differentiated ( $>30$ days). After 21 days of culturing the Caco- 2 cells form well-developed tight junctions. The tight junctions are discrete regions of membrane apposition between adjacent epithelial cells at the luminal aspect of the apical junction complex (demonstrated by transmission electron microscopy) forming a selectively permeable barrier and are the rate-limiting step in the paracellular pathway. Freeze fracture electron microscopy then shows the tight junction to be a series of anastomosing stands. Although previous models of the tight junction considered it to have static permeability properties, more recent research has shown it to be a dynamic structure with the ability to alter its permeability in response to extracellular stimuli (9).

\section{Growth and maintenance of Caco-2 cells}

Caco-2 can be obtained from the American Type Culture Collection (ATCC) or from European Collection of Cell Cultures (ECACC). They are generally grown in serum containing cell culture medium (DMEM) in normal tissue culture flasks. A special feature of Caco-2 cells is that they attached strongly to the plastic of the tissue culture flasks (4). For the transport studies they are seeded on permeable cell culture inserts with the pore diameter $(0.4 \mu \mathrm{m})$ to avoid transmigration of Caco- 2 cells from the apical to the basolateral side. The seeding density is important because it determines the time to reach confluence and subsequent differentiation. The seeding density has been shown to af fect the morphology of the Caco- 2 cell monolayers as well as expression of some transporters (18). Also the number of passage and various culture conditions can influence the expression of the transporters (21). The conventional culture period of Caco-2 cells for transport studies is 21 days, judging from membrane integrity as assessed by measuring (i) transepithelial electrical resistance (TEER), (ii) permeability of hydrophilic paracellular markers (mannitol), (iii) permeability of lucifer yellow or phenol red.

\section{Transport of drugs in Caco-2 monolayer and intestinal tissues}

Oral administration of drugs is the route of choice for drug delivery (11). Oral drug absorption is influenced by many factors besides drug permeability, such as drug solubility, dissolution, active transport and, in some cases, presystemic metabolism (6). In order to be absorbed, a drug has to diffuse across a series of separate barriers. These include from the mucosal side, the mucus gel layer, the intestinal epithelial cells, the lamina propria and the endothelium of the capillaries. Among these, the single layer of epithelial cells is the most significant barrier to drug absorption (3). It is therefore possible to use monolayer of the intestinal epithelial cells to study drug absorption. Drug absorption across the intestinal membrane is a complex process and may occur by one or more of four different routes: the passive transcellular and paracellular routes, the carrier mediated route and by transcytosis (5). Intestinal epithelium presents a major barrier to orally administered drugs.

Caco-2 monolayers have been used to study drug transport by all four routes.

\section{Passive transcellular and paracellular transport}

Passive diffusion driven by concentration gradient occurs through transcellular (via the cell membrane of enterocytes) or paracellular (via the tight junction between the enterocytes) pathways (Fig. 1). Lipophilic compounds can rapidly partition from the luminal fluid into the cell membranes of the intestinal epithelium and are mainly transported via transcellular pathway. Presence of the highly specialized structure (tight junctions) in the intercellular space restricts the free passage of compounds traversing the intestinal epithelium via the paracellular process. Hence, only hydrophilic compounds of low molecular weight (e.g., mannitol) and peptides partitionated poorly into the cell membranes cross the intestinal epithelium via the paracellular route (22). The paracellular transport, involving passive diffusion of compounds in aqueous solution, is less efficient than transcellular process because of the much lower surface area available to the compounds entering the intercellular space (7).

\section{Carrier-mediated transport}

Apart from passive diffusion, active or passive (facilitated diffusion) carrier-mediated transport (Fig. 1) of certain nutrients, vitamins and their structural analogues within a series of xenobiotics occur by specific transporters/carriers present in intestinal epithelium (shown in Fig. 2). Drug transporters can be divided into two major classes: uptake and efflux transporters (Fig. 1) and are localized in the intestine both on the apical side and basolateral membrane. These uptake and efflux transporters belong to two major transporter superfamilies: the ATP-binding cassette $(\mathrm{ABC})$ and solute carrier (SLC and SLCO) family. The major uptake transporters belong to SLC and SLCO superfamilies (e.g., peptide transporter (PEPT), organic anion transporter (OAT), organic anion transporting polypeptide (OATP), organic cation transporter (OCT)) and these transporters do not utilize energy from ATP and transport the drugs according to their concentration gra- 


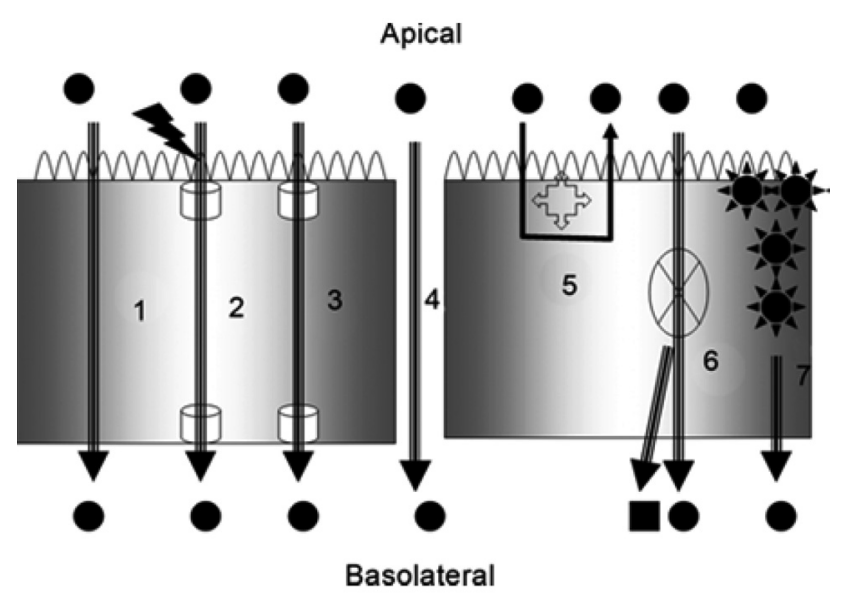

Fig. 1: Multiple pathways for intestinal absorption of a compound: (1) passive, transcellular; (2) active, carrier-mediated; (3) passive, carrier-mediated (facilitated diffusion); (4) passive, paracellular; (5) efflux transporters; (6) intestinal first-pass metabolism followed by absorption of parent and metabolite; and (7) receptor-mediated transcytosis (in Balimane et al. (7)).

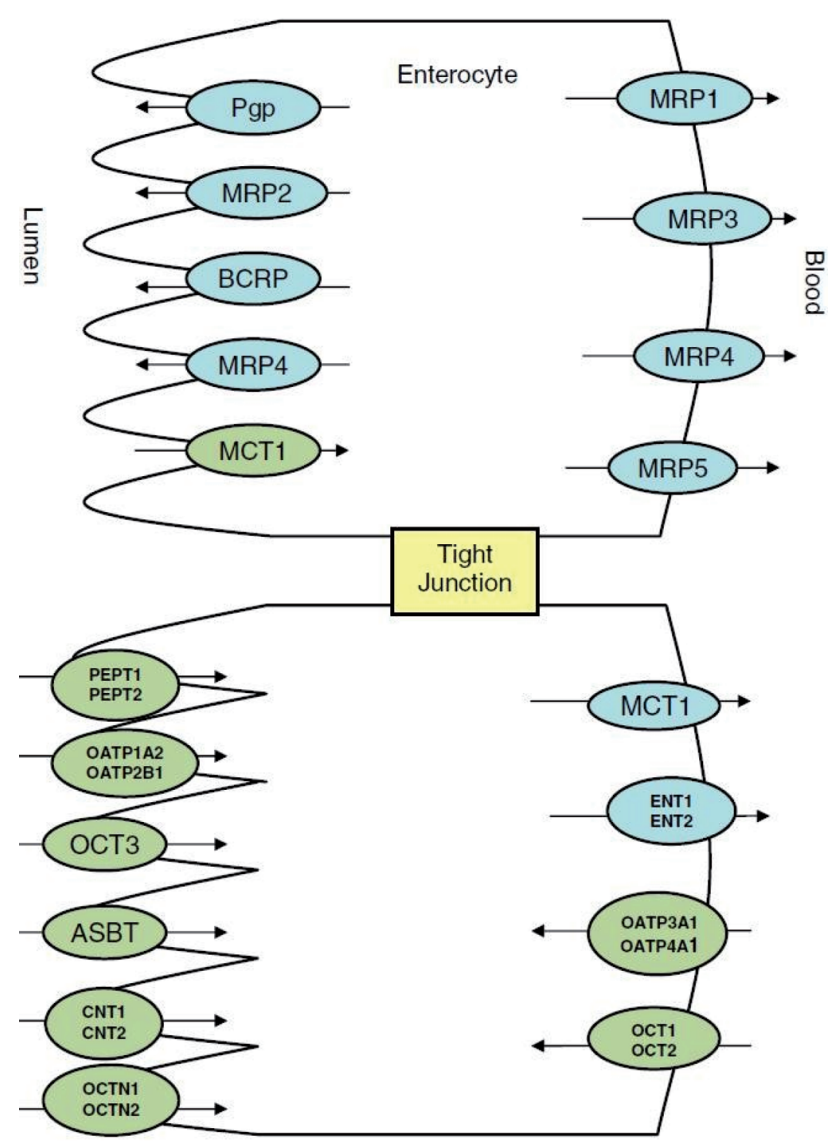

Fig. 2: Various transporters, both efflux and uptake, that are expressed in the intestine (27) both on the apical side (lumen) and on the basolateral membrane (blood). dient. Efflux transporters (Fig. 1) expressed in the intestine include P-glycoprotein (P-gp), multidrug resistance proteins (MRP 1-6), and breast cancer resistance protein (BCRP), are all member of the ATP-binding cassette $(\mathrm{ABC})$ which use ATP as an energy source, allowing them to pump substrates against a concentration gradient $(20,27,25)$ from the cytoplasm of intestinal cells back to the intestinal lumen or to blood. P-glycoprotein, the product of the MDR1 gene, is the most studied member of the apical efflux transporters and has been shown to limit intestinal absorption of a large number of drugs.

Expression of the various transporters in Caco- 2 cells is extensively studied and is influenced by a lot of factors such as: origin of the cells, number of passage, culture conditions and seeding density (21).

\section{Transcytosis}

Certain highly potent macromolecular drugs are transported via receptor-mediated vesicular transport, e.g. transcytosis (Fig. 1). Low transport capacity and the presence of proteolytic enzymes in membrane vesicles limit this pathway as a general drug transport route $(22,26)$.

Transport is often mediated partly by the carrier and partly by passive routes. Since carrier-mediated transport is saturable, the contribution of the passive route will increase with increasing dose. If the drug has a low passive permeability, saturation of the carrier will result in a decreased absorbed fraction (34). In general, passive diffusion is the main mechanism for absorption of many lipophilic compounds, while the carrier-mediated process governs the absorption of transporter substrates.

\section{Caco-2 cells experiment}

Caco- 2 cells (ECACC) are cultured in a standard manner $(8,28,29)$ in plastic tissue culture flasks in DMEM at $37^{\circ} \mathrm{C}$ in an atmosphere of $5 \% \mathrm{CO}_{2}$ and $90 \%$ relative humidity.

\section{Cell viability}

Cell viability is determined by Trypan blue exclusion method (24), i.e. Caco-2 cells are seeded onto Petri dishes (area $9.2 \mathrm{~cm}^{2}$ ) at a density of $2 \times 10^{5}$ cells $/ \mathrm{cm}^{2}$ and cultured for $24 \mathrm{~h}$. Tested drug or transport medium (as a control) is then added and incubated for $2 \mathrm{~h}$. Then the cells are released from the dishes and incubated with $0.4 \%$ Trypan blue for $5 \mathrm{~min}$ and counted by using microscopy.

\section{Assessment of monolayer integrity}

Before the start of the experiment, the monolayer integrity is checked by $500 \mu \mathrm{mol} / 1$ phenol red permeability/ $1 \mathrm{~h}$ $(15,17)$. During the transport studies, the integrity is confirmed simultaneously by ${ }^{14} \mathrm{C}$-mannitol $(0.5 \mu \mathrm{Ci} / \mathrm{ml})$ permeability (4).

\section{Transport studies}

For transport studies, Caco-2 cells are seeded onto the Transwell inserts with semipermeable membrane (Fig. 3) at 
a density of $2.5 \times 10^{5}$ cells $/ \mathrm{cm}^{2}$ and grown to late confluence (21-24 days) replacing the tissue culture medium three times a week and during the last week every day.

Transport experiments are performed as previously described $(28,29,32)$. At the beginning of the experiment, the inserts are rinsed twice and equilibrated with prewarm transport medium at $37{ }^{\circ} \mathrm{C}$ for $30 \mathrm{~min}$. The transport APBL (Fig. 4) experiments are initiated by replacing the transport medium with the diluted drug in the transport medium on the apical side. The samples from the basolateral compartment are withdrawn at 30,60,90 and $120 \mathrm{~min}$ for HPLC analysis or liquid scintillation spectrometry. Similarly, for BL-AP transport, the drug is added to the basolateral compartment and samples from the apical compartment withdrawn in the same time intervals.

Influence of $\mathrm{pH}$ gradient ( $\mathrm{pH}$ apical 6/ basolateral 7.4) can be also studied. To determine paracellular component of the drug transport, the $\mathrm{Ca}^{2+}$ - free transport medium is used. During the inhibition studies the inhibitor of the certain transporter (e.g., verapamil, probenecid) is administered together with the studied drug.

\section{Estimated parameters}

At the end of the experiment, the apparent permeability coefficient $\mathrm{P}_{\mathrm{app}}(\mathrm{cm} / \mathrm{s})$ is calculated according to Artursson (3): $\mathbf{P}_{\text {app }}=(\mathbf{d} Q / \mathbf{d} t) \times\left(\mathbf{1} / \mathbf{A} \times \mathbf{C}_{\mathbf{0}}\right)$, where $\mathrm{d} Q / \mathrm{d} t$ is the permeability rate, the amount of drug appearing in the re-
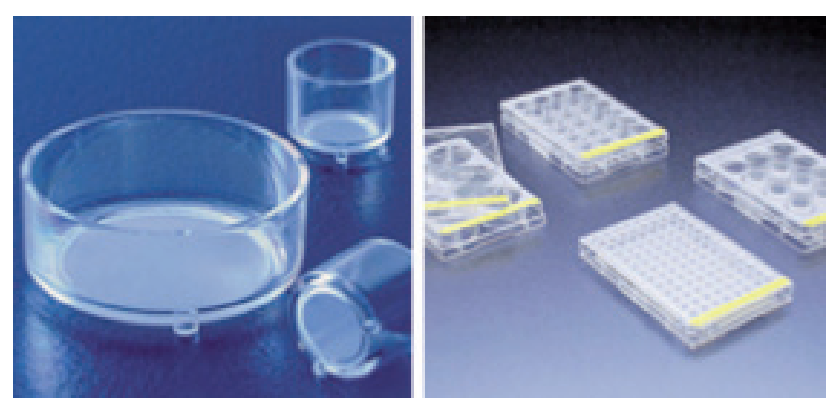

Fig. 3: Transwell inserts with semipermeable membrane and desks for transport studies.

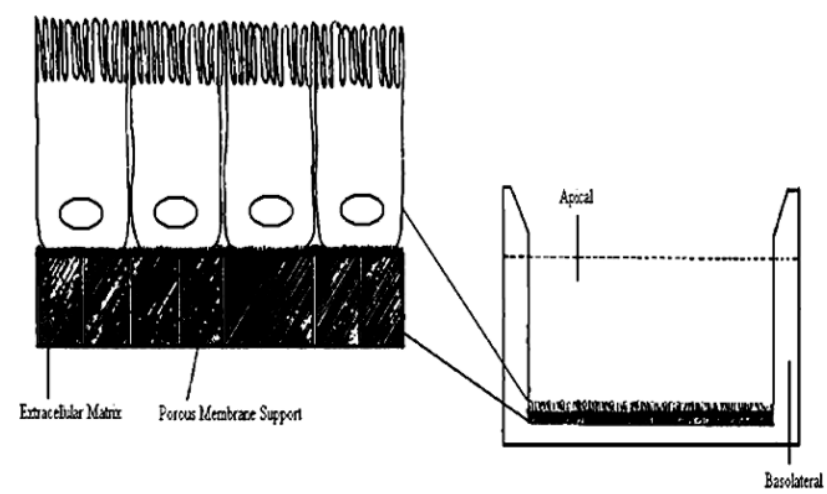

Fig. 4: Schematic representation of a cell monolayer grown on a permeable support (26). ceiver compartment in function of time $(\mathrm{nmol} / \mathrm{s}), \mathrm{C}_{0}$ is the initial concentration in the donor chamber $(\mathrm{nmol} / \mathrm{ml})$, and $A$ is the surface area of the monolayer $\left(\mathrm{cm}^{2}\right)$.

Transport in both directions across monolayer enables to calculate (30) $\mathbf{P}_{\text {app }}$ uptake ratio $=\mathbf{P}_{\text {app }}$ AP-BL $/ \mathbf{P}_{\text {app }}$ BL-AP showing if the transport is passive or active.

\section{The Biopharmaceutics Classification System (BCS)}

A majority of drugs marketed worldwide are administered orally. The efficacy of these drugs is dependent on the oral bioavailability, which in turn, is dependent on extent of absorption. Oral absorption, in basic terms, is dependent on the intestinal drug solubilization and the intestinal drug permeability. The amount of drug that goes into solution is affected by factors such as volume, $\mathrm{pH}$, temperature and the compound's octanol/water partition coefficient. The amount of drug that crosses the membrane is affected by factors such as concentration, temperature, time, surface area, viscosity, and affinity to a membrane transporter. Mechanistically, the permeability of a drug is additively determined by a number of parallel processes such as a passive component and one or more active components (10). The good correlation between permeability across Caco-2 cell monolayer, solubility and oral bioavailability in humans has been demonstrated (30).

Amidon et al. (2) recognized the fundamental parameters controlling the rate and extent of oral drug absorption and devised Biopharmaceutics Classification System (BCS) that categorized drugs into four classes according to their solubility and permeability (Fig. 5). The objective of the $\mathrm{BCS}$ is to predict in vivo pharmacokinetic performance of drug products from measurements of permeability and solubility (2).

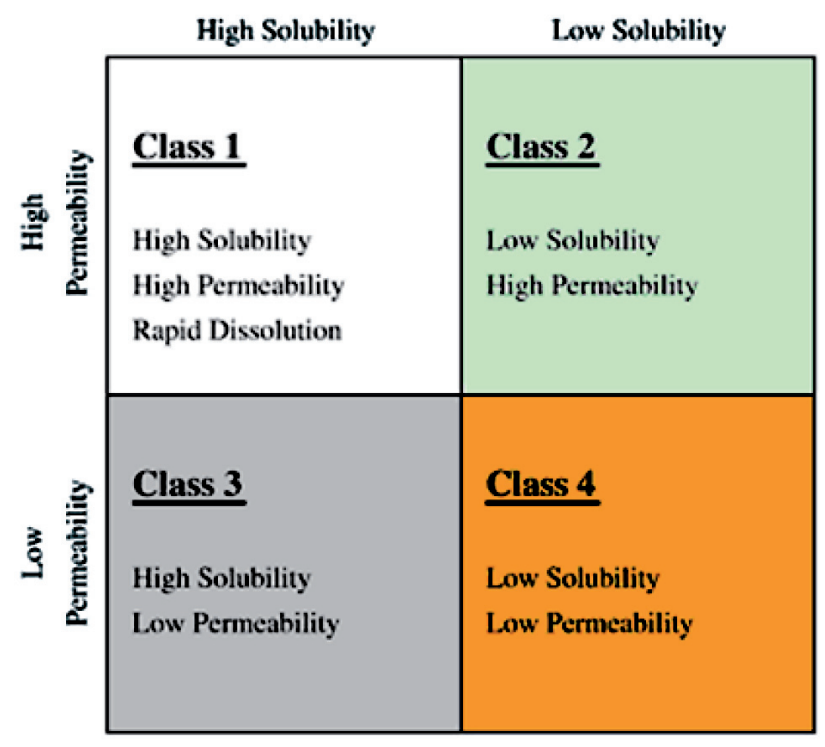

Fig. 5: The Biopharmaceutics Classification System (BCS) as defined by Amidon et al. (2). 
As depicted in Fig. 5, the BCS sorts drugs on a scale in terms of solubility versus permeability (10). A drug substance is considered "highly soluble" when the highest marketed dose strength is soluble in $250 \mathrm{ml}$ of aqueous media over a $\mathrm{pH}$ range of $1-6.8$ at $37{ }^{\circ} \mathrm{C}(13,33)$. A drug substance is considered to be "highly permeable" when the extent of absorption in humans is determined to be greater or equal to $85 \%$ of an administered dose $(13,33)$. Accordingly, Class 1 compounds possess high properties of both solubility and permeability (e.g., diazepam, propranolol), while Class 4 compound possess low properties (e.g., sulfasalazine); Class 2 are highly permeable and poorly soluble (e.g., ibuprofen, carbamazepine), while Class 3 possess the opposite characteristics of poor permeability and high solubility (e.g., atenolol, metformin) (33).

\section{The BCS-based biowaiver approach}

The scientific concept of the BCS (2) was incorporated in the regulation of the USA (Guidance for Industry. Waiver of In Vivo Bioavailability and Bioequivalence Studies for Immediate-Release Solid Oral Dosage Forms Based on a Biopharmaceutics Classification System; (14)), in the Europena Union regulation (EMEA - Committee for medical products for human use CHMP - BCS-based biowaiver; (13)) and also in the World Health Organization (WHO) „Proposal to waive in vivo bioequivalence requirements for WHO Model of Essential Medicines immediate-release, solid oral dosage forms" (33).

The BCS-based biowaiver approach is meant to reduce in vivo bioequivalence studies, i.e., it may represent a surrogate for in vivo bioequivalence. The term "biowaiver" is defined by the WHO as "approval of generic solid oral formulation of an active pharmaceutical ingredient based on strictly defined dissolution criteria as a surrogate for an in vivo bioequivalence test".

The principles may be used to establish bioequivalence in applications for generic medicinal products, extensions of innovator products, variations that require bioequivalence testing, and between early clinical trial products and tobe-marketed products. This will certainly reduce costs and time in the drug development process and reduce unnecessary drug exposure in healthy subjects, which is normally the study population in bioequivalence studies. The BCS is today only intended for oral immediate-release products that are absorbed throughout the intestinal tract (23). To be considered bioequivalent according to the biowaiver procedure, a pharmaceutical product: should contain a BCS class 1 compound; should be rapidly dissolving ( $\geq 85 \%$ in 15 minutes in $\mathrm{pH}$ 1.2-6.8); should not contain excipients which could influence the absorption of the compound; should not contain an compound with a narrow therapeutic index; and should not be designed to be absorbed from the oral cavity (13). The WHO guidance (33) not only allows biowaiver of the drug product containing drug from BCS Class 1, but also opens the possibility for drug pro- ducts containnig compounds from BCS 3 and certain compounds from BCS Class 2 to qualify for biowaiver-based approval.

The data submitted for the first successful BCS-based application in Germany (for sotalol hydrochloride) were published by Alt et al. (1).

\section{Conclusion}

Caco-2 cells are widely acceptable in vitro tool for studying drug transport across the intestinal. The transport of drugs across the intestinal epithelium may occur by one or more of four different routes: the passive transcellular and paracellular route, the carrier mediated route and by transcytosis. In general, passive diffusion is the main mechanism for absorption of many lipophilic compounds, while the carrier-mediated process governs the absorption of transporter substrates. The knowledge of the drug permeability together with knowledge of its solubility enables to rank the drug according to Biopharmaceutics Classification System (BCS). For certain drugs, the BCS-based biowaiver approach can be used to reduce in vivo bioequivalence studies (for generic medicinal products, extensions of innovator products, variations that require bioequivalence testing, and between early clinical trial products and to-be-marketed products). This will certainly reduce costs and time in the drug development process and reduce unnecessary drug exposure in healthy subjects, the study population in bioequivalence studies.

\section{Acknowledgements}

The work was supported by the research project GAČR $305 / 08 / 0535$.

\section{References}

1. Alt A, Potthast H, Moessinger J, Sickmüller B, Oeser H. Biopharmaceutical characterization of sotalol-containing oral immediate release drug products. Eur J Pharm Biopharm. 2004;58:145-50.

2. Amidon GJ, Lennernäs H, Shap VP, Crison JR. A theoretical basis for a biopharmaceutic drug classification: the correlation of in vitro drug product dissolution and in vivo bioavailability. Pharm Res. 1995;12:413-20.

3. Artursson P, Karlsson J. Correlation between oral drug absorption in humans and apparent drug permeability coefficients in human intestinal epithelial (Caco-2) cells. Biochem Biophys Res Comm. 1991;175:880-5.

4. Artursson P, Karlsson J, Ocklind G, Schipper N. Studying transport process in absorptive epithelia. In: Shaw AJ, editor. Epithelial cell culture - a practical approach, Oxford University Press, 1996:111-33.

5. Artursson P, Palm K, Luthman K. Caco-2 monolayers in experimental and theoretical predictions of drug transport. Adv Drug Deliv Rev. 2001;46:27-43.

6. Artursson P, Tavelin S. Caco-2 and emerging alternatives for prediction of intestinal drug transport: a general overview. In: H. van de Waterbeemd, H. Lennernas and P. Artursson, Editors, Drug Bioavailability: Estimation of Solubility, Permeability, Absorption and Bioavailability, Wiley-VCH Verlag GmbH \& Co. KgaA, Weinheim, 2003:72-83.

7. Balimane PV, Han $\mathrm{YH}$, Chong S. Current industrial practices of assessing permeability and P-glycoprotein interaction. AAPS J. 2006;8:E1-13

8. Bourdet DL, Thakker DR. Saturable absorptive transport of the hydrophilic organic cation ranitidine in Caco-2 cells: role of $\mathrm{pH}$-dependent organic cation uptake system and P-glycoprotein. Pharm Res. 2006;23:1165-77.

9. Clayburgh DR, Shen L, Turner JR. A Porous Defense: the Leaky Epithelial Barrier in Intestinal Disease. Laboratory Investigation 2004;84:282-91.

10. Custodio JM, Wu CY, Benet LZ. Predicting drug disposition, absorption/elimination/transporter interplay and the role of food on drug absorption. Adv Drug Deliv Rev. 2008;60:717-33 
11. Delie F, Rubas W. A human colonic cell line sharing similarities with enterocytes as a model to examine oral absorption: advantages and limitations of the Caco-2 model. Crit Rev Ther Drug Carrier Syst. 1997;14:221-86.

12. Doherty MM, Charman WN. The mucosa of the small intestine: how clinically relevant as an organ of drug metabolism? Clin Pharmacokinet. 2002;41:235-53.

13. EMEA. European Medicines Agency: Guideline on the investigation of bioequivalence. Committee for medicinal products for human use (CPMP/EWP/QWP/ 1401/98 Rev. 1/ Corr.), London, January 2010, 25-7. http://www.ema.europa.eu/ pdfs/human/qwp/140198enrev1fin.pdf.

14. FDA. Food and Drug Administration, Guidance for Industry: Waiver of In Vivo Bioavailability and Bioequivalence Studies for Immediate Release Solid Ora Dosage Forms Based on a Biopharmaceutics Classification System, 2000 Retrieved from http://www.fda.gov/downloads/Drugs/GuidanceCompliance RegulatoryInformation/Guidances/UCM070246.pdf.

15. Fleet JC, Wood RJ. Specific $1,25(\mathrm{OH})_{2} \mathrm{D}_{3}$-mediated regulation of transcellula calcium transport in Caco-2 cells. Am J Physiol 1999;276:G958-64.

16. Fossati L, Dechaume R, Hardillier E, et al. Use of simulated intestinal fluid for Caco-2 permeability assay of lipophilic drugs. Int J Pharm. 2008;360:148-55.

17. García-Casal MN, Leets I, Layrisse M. Beta-carotene and inhibitors of iron absorption modify iron uptake by Caco-2 cells. J Nutr. 2000;130:5-9.

18. Hayeshi R, Hilgendorf C, Artursson P, et al. Comparison of drug transporte gene expression and functionality in Caco-2 cells from 10 different laboratories. Eur J Pharm Sci. 2008;35:383-96.

19. Hidalgo IJ, Raub TJ, Borchardt RT. Characterization of the human colon carcinoma cell line (Caco-2) as a model system for intestinal epithelial permeability. Gastroenterology 1989;96:736-49.

20. Ho RH, Kim RB. Transporters and drug therapy: implications for drug disposition and disease. Clin Pharmacol Ther. 2005;78:260-77.

21. Kamiyama E, Sugiyama D, Nakai D, Miura S, Okazaki O. Culture period-dependent change of function and expression of ATP-binding cassette transporters in Caco-2 cells. Drug Metab Dispos. 2009;37:1956-62

22. Lee K, Thakker DR. Saturable transport of H2-antagonists ranitidine and famotidine across Caco-2 cell monolayers. J Pharm Sci. 1999;88:680-7.
23. Lennernäs H, Abrahamsson B. The use of biopharmaceutic classification of drugs in drug discovery and development: current status and future extension. J Pharm Pharmacol. 2005;57:273-85.

24. Lu Y, Heydel JM, Li X, Bratton S, Lindblom T, Radominska-Pandya A. Lithocholic acid decreases expression of UGT2B7 in Caco-2 cells: a potential role for a negative farnesoid X receptor response element. Drug Metab Dispos. 2005; 33:937-46.

25. Oostendorp RL, Beijnen JH, Schellens JH. The biological and clinical role of drug transporters at the intestinal barrier. Cancer Treat Rev. 2009;35:137-47.

26. Shah P, Jogani V, Bagchi T, Misra A. Role of Caco-2 Monolayers in Prediction of Intestinal Drug Absorption. Biotechnol Prog. 2006;22:186-98.

27. Shugarts S, Benet LZ. The role of transporters in the pharmacokinetics of orally administered drugs. Pharm Res. 2009;26:2039-54.

28. Smetanova L, Stetinova V, Kholova D et al. Caco-2 cells and Biopharmaceutics Classification System (BCS) for prediction of transepithelial transport of xenobiotics (model drug: caffeine). Neuro Endocrinol Lett. 2009;30(Suppl. 1):101-5.

29. Stetinova V, Smetanova L, Kholova D et al. Transepithelial transport of ambroxol hydrochloride across human intestinal Caco-2 cell monolayers. Gen Physiol Biophys. 2009;28:309-15.

30. Ungell A-L, Karlsson J. Cell culture in drug discovery: an industrial perspective. In: van de Waterbeemd H, Lennernäs $\mathrm{H}$, Artursson P, editors. Drug bioavailability. Weinheim: Wiley-Vch., 2004:90-131.

31. Vachon PH, Beaulieu J-F. Transient Mosaic Patterns of Morphological and Functional Differentiation in the Caco-2 Cell Line. Gastroenterology 1992; 103:414-23

32. Walle UK, Walle T. Taxol transport by human intestinal epithelial Caco-2 cells. Drug Metab Dispos. 1998;26:343-6.

33. WHO. Proposal to waive in vivo bioequivalence requirements for WHO Mode List of Essential Medicines immediate-release, solid oral dosage forms. Annex 8 , of WHO Expert Committee on Specification for Pharmaceutical Preparations. Geneva: World Health Organization. 2006

34. Yu L, Zeng S. Transport characteristics of zolmitriptan in a human intestinal epithelial cell line Caco-2. J Pharm Pharmacol. 2007;59:655-60.

Received: 30/06/2010.

Accepted in revised form: 06/12/2010.

\section{Corresponding author:}

Libuše Smetanová, MD, Institute of Experimental Biopharmaceutics, Joint Research Centre of PRO.MED.CS Praha a.s. and the Academy of Sciences of the Czech Republic, Heyrovského 1207, 50003 Hradec Králové, Czech Republic; e-mail: smetanova@uebf.cas.cz 